\title{
BIOTECHNOLOGY IN ANIMAL AGRICULTURE
}

I have been asked to assess the interface of biotechnology and animal agriculture, paying particular attention to recent successes and failures, and to identify potential opportunities as well as bottlenecks to further advances. Some aspects of this assignment are relatively straightforward, while others require so many qualifiers as to be of limited value. The first problem is to define biotechnol-

GEORGE E. SEIDEL Jr.

Animal Reproduction and Biotechnology Laboratory Colorado State University Fort Collins, CO 80523

For over twenty years, Seidel has worked to perfect techniques for manipulating embryos. Current projects include procedures to make transgenic animals, in vitro oocyte maturation and fertilization, chemically defined media for culturing embryos and genetic control of maternal recognition of pregnancy. ogy. In a broad sense, every aspect of agriculture is biotechnology, whereas a narrow definition limited to recombinant DNA techniques would show only barely perceptible effects on production agriculture at this time. A broad definition of biotechnology will be used and items of potential interest will be emphasized. Defining animal agriculture is also somewhat arbitrary. Currently, the most important agricultural species in the United States are cattle, swine, sheep, chickens and turkeys. Horses might be considered more recreational than agricultural. A considerable percentage of beef cattle operations also have a huge recreational component, as do those concerning other spe-

cies. Many of the opportunities to apply biotechnology are on farms where the majority of family income is from non-farm sources. There also are biotechnology applications in less common species such as buffalo, deer, goats, ducks, geese and fish.

BRIEF SUMMARY OF THE STATUS OF ANIMAL AGRICULTURE

Currently about two percent of the labor force in the United States is employed in production agriculture, and another 20 percent of the labor force is involved in servicing production agriculture (e.g., fertilizer, farm equipment) or transporting, processing, and marketing agricultural products. Roughly half of these people are in the animal agriculture sector. This 
In a broad

sense, every

aspect of

agriculture

is biotech-

nology...

...unless

American

farmers

become more

efficient, their

jobs will be

replaced by

farmers in

other countries

who export

their produce

to the United

States.

huge enterprise is reasonably successful by most measures, e.g. providing abundant, healthful products at the lowest cost in the world as measured by percentage of median disposable family income used for food (currently 12 percent). This is all the more remarkable because of the huge percentage of food expenditures that go for packaging, storage, processing, safety and quality control, advertising, and service in restaurants and related establishments.

This situation is less rosy when examined in depth; e.g., those involved in production agriculture have not fared well over the years (a robust measure of this is the continuing exodus of people from production agriculture, particularly involving animals). The huge direct and indirect government subsidies to address this problem have met with only marginal success and add indirectly to the cost of food, although these costs are low compared to most other industrialized countries. It also must be made plain that improving efficiency of animal agriculture with biotechnology, while greatly benefitting consumers, will probably harm more farmers than it will help because in our economic system, those who do not adopt more efficient techniques cannot compete with those who do. On the other hand, unless American farmers become more efficient, their jobs will be replaced by farmers in other countries who export their produce to the United States.

\section{BIOTECHNOLOGIES ARE TOOLS}

Agricultural practices are continually refined and perfected. For example, 10.000. 00o dairy cows currently produce considerably more milk than 25.000. ooo did in 1945; in 1990 beef production from calves of 33,000,000 beef cows exceeded that of 45,000,000 cows in 1975. Similar successes have been recorded for poultry and swine. There are huge opportunities to improve this performance further, particularly with cattle, since the best herds produce twice the output per animal as the average herd. Continued improvements arise from dozens of sources, each of which only contributes modestly. An excellent example is selective breeding. Although the improvement with each generation is modest, it accumulates like compound interest. In most cases, new biotechnologies should be thought of as tools to aid this process. They face stiff competition from current tools, which already are very effective. Any compromise of currently effective tools must be compensated by extraordinary benefits, or the new technol- 
ogy will not even be considered. A good example is introducing a transgene for disease resistance into a breeding population of dairy cows. Unless the transgene is already in a high milk-producing line, the economic costs of lowered milk production in the initial generations would cancel the benefits of controlling any known disease.

\section{RECENT BIOTECHNOLOGICAL BREAKTHROUGHS}

I have constructed three lists of biotechnologies used in production animal agriculture (Tables 1-3). The first list, technologies commercialized before 1980 (Table 1), might be considered inappropriate for the objectives of this paper. Nevertheless, I present them because they are of overriding

TABLE 1 EXAMPLES OF BIOTECHNOLOGIES IN WIDESPREAD USE COMMERCIALIZED BEFORE 1980

Selective breeding

Nutrient requirements of animals

Feed analysis

Vaccination

Veterinary diagnostics and therapeutics

Artificial insemination (mostly cattle and poultry)

Crossbreeding

Regulation of reproductive cycles

Embryo transfer

Ultrasound to measure carcass fat

importance in current animal agriculture, they illustrate the kinds of technologies that work, and some of them just came into widespread use around 1980, e.g., regulation of reproductive cycles and embryo transfer technology. It is important to recognize that most technologies are applied directly only to a minority of animals in the population. Some of these niches only apply to specialty markets, e.g., embryo transfer to introduce new germ plasm into specific pathogen-free swine herds, while others filter down to the whole population, e.g., artificial insemination is only done with five percent of beef cattle, but over half of the bulls that breed beef cattle via natural service are conceived by artificial insemination or had parents conceived by artificial insemination. Some biotechnologies apply to every individual in the population. For example, all tur- 
keys are conceived by artificial insemination because males have so much breast meat that it interferes physically with natural mating.

The second list, biotechnologies commercialized in the 1980s, (Table 2) similarly has technologies widely applied, e.g., growth promotants for growing beef cattle, and those used in narrow niches, e.g., storage of bovine embryos in liquid nitrogen. This latter technology resulted in an entirely new commodity, frozen embryos. Many frozen embryos are imported and exported; a very important fringe benefit of this approach is the greatly decreased danger of spreading diseases compared to importing live animals or semen.

\section{TABLE 2 EXAMPLES OF BIOTECHNOLOGIES IN WIDESPREAD USE COMMERCIALIZED AFTER I980}

Growth promotants (other than DES) and ionophores

Use of bypass (of rumen) protein and fat

Monoclonal antibodies for diagnostics

Storage of embryos in liquid nitrogen

Ivermectin to treat parasites

Ultrasound for veterinary diagnostic purposes

Ivermectin is truly a miracle drug in terms of safety and efficacy. Although this drug was developed for animals, and there is no use for it in people in the United States, it will be one of the most important drugs ever developed for human use in countries where people are regularly infected with certain deadly or debilitating parasites, e.g., the organism causing river blindness in Africa.

The third list, (Table 3) technologies just being commercialized at this time, will be discussed item by item. The list is headed by bovine somatotropin (a growth hormone), a truly marvelous product of biotechnology produced by bacteria via recombinant DNA technology and administered to dairy cows by injection or implant about every two weeks. This treatment increases milk production about 20 percent compared to controls. For sound reasons, it is not administered during the first quarter of lactation, so the increase in milk production is closer to 10 percent over the lifetime of the cow. The main action of this protein hormone in lactating cows is simple: it is a so-called partitioning agent, causing the nutrients that a cow eats to go to milk production rather than carcass fat. With- 
out exogenous somatotropin milk production declines markedly in many

This product cows after the first third of lactation, and many of the nutrients they eat end up as body fat. Somatotropin has other effects too, e.g., it stimulates appetite so cows eat more, thus further increasing milk production.

This product is safe, efficacious, cost-effective, and does not alter milk composition, yet it is one of the most controversial products ever developed. One problem is current milk surpluses; another is that it is a recombinant DNA product. However, it is unlikely that even a few percent of recombinant DNA products will be able to come close to the safety and efficacy parameters of this product; it should be a great cause of concern that this product is perceived so negatively by so many.

At this writing there is a glut of crude oil on the world market, but think of the benefits of 10 percent increase in fuel efficiency of internal combustion engines. There would be costs too, less employment in oil fields just as fewer dairy cows and dairy farmers will be needed after bovine somatotropin comes into use. However, precisely these kinds of efficiency are required for the American dairy farmer to compete; also, a lower number of cows equates to less pollution, less grain consumption, etc.

TABLE 3 EXAMPLES OF BIOTECHNOLOGIES BEGINNING TO BE USED

COMMERIC ALLY IN THE EARLY 1990S

Bovine somatotropins to improve milk production

Vaccines inducing antibodies distinguishable from those due to natural infections

Cloning embryos by nuclear transplantation

Sexing embryos

In vitro maturation of oocytes from slaughterhouse ovaries

In vitro fertilization and culture of embryos

Oral melatonin to control seasonal breeding in sheep and goats

Induced twinning in cattle

The second item in Table 3 is a solution to an old problem, distinguishing healthy animals with antibodies due to vaccination from animals making antibodies due to current infection with a contagious agent. A well-known example is bovine brucellosis, which causes great economic loss due to abortion. This organism also is a threat to people; it causes undulant fever, which usually cannot be treated effectively, and is very de- 
...making ex-

act genetic

copies of

outstanding

animals by

nuclear trans-

plantation has

been a dream

of animal

breeders for

years. bilitating for years. The program to eradicate brucellosis in cattle has been extremely expensive and hindered by the above problem. However anew vaccine developed with recombinant DNA techniques is about to be released that elicits antibodies distinguishable from those induced by the brucella organism itself. A similar vaccine has been developed for pseudorabies in swine.

The third item in Table 3, making exact genetic copies of outstanding animals by nuclear transplantation has been a dream of animal breeders for years. This now can be accomplished by combining serial cloning of embryos (e.g., transplanting nuclei from a 16-cell embryo into 16 unfertilized oocytes, and when they reach the 16-cell stage, repeating the process) and cryo-preservation of embryos so that a genetic copy in embryo form is available after other cloned embryos become adults. If the adults are outstanding, copies can be made; if not, the embryos are discarded and other clones propagated.This technology has other advantages: automatic sex selection, faithful reproduction of heterozygous transgenic animals, and reproduction of outstanding crossbred animals. Note that without this technology these latter two types of animals do not breed true, much like the situation with hybrid corn.

Item four is sexing embryos. Although still a bit clumsy, commercial sexing of bovine embryos with a Y-chromosome-specific DNA probe is now offered commercially. This technology eventually will be replaced by sexed semen. The fifth item in Table 3 , in vitro maturation of oocytes from slaughterhouse ovaries, is now becoming sufficiently reliable so that we can exploit this source of genetic material via in vitro fertilization and embryo transfer. Because costs per animal for embryo transfer remain high, most applications will be in cattle for the next decade (a superior calf is worth much more than a superior lamb). Two properties of harvesting female gametes in this way are noteworthy. The first is reproduction from a dead animal. Thus a valuable cow or horse that dies or is slaughtered due to terminal illness can reproduce; note that this procedure works at any stage of the reproductive cycle and even during pregnancy. Another advantage is to exploit genes of slaughtered females with especially good carcasses such as high dressing percentage, tender meat with minimal fat, etc.

The second noteworthy property of embryos produced in this way is that they can be produced in huge quantities at low cost. This makes them 
appropriate for applications such as twinning cattle, and for other biotechnologies like cloning by nuclear transplantation. This also makes them ideal for many experimental purposes. A special fringe benefit is that experiments can be done without using experimental animals, just nearly worthless ovaries from commercially slaughtered cows plus frozen semen.

In vitro fertilization and culture of embryos are required to exploit many applications of in vitro oocyte maturation, but there are some specific uses of these technologies in addition. For example, in vitro fertilization can be used with technologies of sexing semen that produce too few sperm for artificial insemination but plenty for in vitro fertilization. In vitro fertilization also can be used for testing fertility of males, which is extremely costly and frequently inaccurate with other procedures.

The second from last item in Table 3 concerns melatonin. Sheep and goats are seasonal breeders, which causes great constraints in agricultural productivity. They normally become pregnant when days become short and the nights, long. The pineal gland at the base of the brain secretes melatonin when it is dark and fails to do so in daylight; moreover, melatonin is a key regulator of reproductive hormones in these species. Therefore, one can cause the same effects as darkness by feeding melatonin. A feeding at 4 p.m. is the equivalent of darkness commencing at that time. After a few weeks of this animals resume fertile reproductive cycles and if mated, become pregnant, no matter what the season of the year.

The last item in Table 3, twinning in cattle, can be induced in several ways, including embryo transfer or injections to increase double ovulations. These procedures are just becoming economically feasible because of combining some of the technologies just described. The main principle that makes twinning attractive is that about 70 percent of the nutrients that a beef cow eats in the course of a year go for her maintenance requirements, and the remaining 30 percent go to needs of pregnancy and lactation. Thus for 30 percent more feed, one can produce two calves instead of one. There is, however, higher morbidity and mortality with twins, and more labor is required.

PROMISING BIOTECHNOLOGIES NOT YET COMMERCIALIZED

In addition to standard recombinant DNA procedures, there are numerous extremely powerful biotechnologies being used in the laboratory that 
...although

transgenesis is

one of the most

powerful tech-

nologies avail-

able for

scientific en-

deavors, it is

not likely to

have much

impact in

production

agriculture

over the next

decade.

will eventually impact on animal agriculture. A somewhat arbitrary list is in Table 4. A few of these items will be discussed further. The first, transgenic procedures, is covered in more detail elsewhere in these proceedings. I want to emphasize that although transgenesis is one of the most powerful technologies available for scientific endeavors, it is not likely to have much impact in production agriculture over the next decade. By far the main problem is that we know so little about gene function for production traits in farm animals that we simply do not know what genes to manipulate, much less how to manipulate them. This clearly will change eventually, and although there may be several spectacular applications before the year 2000, most will occur after that date. Other problems with transgenic technology include expense and the extremely long time-frame involved with species with long generation intervals. Sexing semen is an-

TABLE 4 EXAMPLES OF FINDINGS OR BIOTECHNOLOGIES THAT LIKELY WILL HAVE APPLICATION IN PRODUCTION AGRICULTURE IN THE FUTURE

Transgenic procedures

Polymerase chain reaction

Sexing semen

Embryonic stem cells

Homologous recombination

Artificial chromosomes

Somatotropin and beta agonists for meat production

Growth factors

Second messenger systems

Transcription factors and other regulators of gene expression

Early pregnancy factor

Trophoblast-specific interferons

Marker-assisted selection

other promising biotechnology. At least one method of sexing semen, that of using a flow cytometer, has been convincingly demonstrated to sort $\mathrm{X}$ and $\mathrm{Y}$ chromosome-bearing sperm of several species with 80-90 percent accuracy. While the sperm are damaged somewhat, and the procedure is much too slow to be used for routine artificial insemination, the sperm are reasonably fertile and could be used for in vitro fertilization. There is a huge and immediate market for sexed semen in cattle. 
These fall into the category of important areas of research concerning regulation of cellular function; thousands of research papers are published in these areas annually, and the resulting information will be exceedingly useful in getting cells to do useful things. A decade from now, some of these will reach the interface with production agriculture with very important consequences. However, much more information is needed to make educated guesses concerning exactly which factors will be matched with which applications.

Extolling the virtues of marker-assisted selection is currently very much in fashion. Related procedures using restriction fragment length polymorphisms (RFLP) form much of the basis for projects on mapping (not sequencing) human and other genomes. Similar procedures have been successfully applied to finding genes responsible for several human diseases. An example of how this concept works is that one tries to match up specific and easily measured genetic differences (e.g., a particular RFLP) with a desirable characteristic (e.g., a high percentage of unsaturated fat in meat) so as to quickly and easily identify the genetically superior animals in the population. A fringe benefit is that one can further exploit such matchups to locate and identify the genes responsible for the desired characteristic.

This important biotechnology has many potential uses. Unfortunately, there are also severe limitations because dozens of genes are responsible for most traits of interest (e.g., fertility, growth rate, disease resistance), which frequently results in confusion with this approach. However, in other instances, especially where single genes with multiple alleles have huge effects, this approach is excellent.

\section{BIOTECHNOLOGY NEEDS IN ANIMAL AGRICULTURE}

If one asked about biotechnological needs, one likely would get quite different answers from different groups, e.g., farmers vs animal scientists. I have listed some of the more obvious ones in Table 5. Many of these have been alluded to earlier; the usefulness of most is obvious. A number of those listed would result in markets in excess of $\$ 100,000,000$ annually. Some would lead to entirely new approaches and greatly increase efficiency, e.g., cloning animals from adult cells.

New biotechnologies as well as new applications of older biotechnologies continue to be applied to increase the efficiency of animal agriculture. 
All of these organisms are more complicated than supercomputers or space ships.
TABLE 5 BIOTECHNOLOGICAL NEEDS IN ANIMAL AGRICULTURE

Gene maps

Basic information about all aspects of animal biology, especially appetite, stress, disease

Sexed semen (eggs for poultry)

Improved techniques to cryopreserve poultry semen

Cloning animals from adult cells

Reliable and simple transgenic technology

Early pregnancy tests

In vitro gametogenesis

Methods of modifying animal products for fat and other characteristics

Quality control for salmonella, etc.

Very inexpensive diagnostics for on-farm use

Immunocastration

Reprogramming laying hens

BOTTLENECKS TO APPLYING BIOTECHNOLOGY TO

ANIMAL AGRICULTURE

Some obvious bottlenecks are listed in Table 6. Interestingly, they fall into two broad categories: biological constraints and sociological/societal constraints. Farm animals are much more complex than single cell organisms. All of these organisms are more complicated than super computers or space ships. Therefore it takes enormous resources to understand and manipulate them to useful ends, including modifying organisms genetically. However, there is considerable optimism in scientific circles in dealing with this obstacle. It is considered challenging, interesting, and important, and progress is being made. The complexity of cells and organisms is truly amazing. Even non-cellular entities such as viruses are complex, and their interaction with cells especially complex. A graphic example is the human immunodeficiency (AIDS) virus; direct and indirect funding to understand this virus is approaching one billion dollars annually on a world-wide basis, and while information is accumulating exponentially, many questions remain concerning effective means of controlling this virus other than by human behavioral modifications. Note also that one billion dollars is really minuscule compared to military expenditures.

The other items in Table 6 are also bottlenecks, some of them diffuse ones. Any one or two of these might be dealt with easily, but their cumulative effect can be devastating. For example, fewer students are undertaking 
Complexity of organisms

Diffuse, underfunded efforts

Instability of funding

Secrecy due to proprietary considerations with many sources of funding

Research climate that discourages young scientists

Costs of FDA approval process

Regulatory costs of doing research

Time required to prepare proposals, get committee approvals, prepare progress reports, etc.

Narrow training and experience of most life scientists

Lack of clear goals

Remoteness of many scientists from needs of farmers

graduate studies in animal biology than in the past, which perhaps is desirable since employment opportunities are limited. Another very interesting phenomenon is the change from predominantly men to predominantly women graduate students in these areas. It appears that fewer young men find this area of endeavor attractive in the United States, at least in part due to some of the items in Table 6.

Despite the cumulative insidiousness of the items in Table 6, many are desirable and necessary, and most alternatives to them are even less desirable. Thus, the solution is to deal with the items in Table 6, even though this takes huge amounts of time and funding.

\section{SUMMARY}

Hundreds of biotechnologies are applied daily in animal agriculture in the United States. New biotechnologies as well as new applications of older biotechnologies continue to be applied to increase the efficiency of animal agriculture. At the same time, new and more varied animal products are being produced. Because there are considerable time lags between conceiving a new application and its use in production agriculture, it is too early to evaluate eventual impacts of newer technologies such as recombinant DNA procedures. However, it is likely that they will be exceedingly important for animal agriculture in the decades ahead. Although there are considerable regulatory and safety costs in applying biotechnology, by and large, these costs are reasonable and need to be taken into account in planning and budgeting. 


\section{SELECTED REFERENCES}

Evans, J.W. and A.Hollaender, eds. 1986. Genetic Engineering of Animals: An Agricultural Perspective. 328 pp. Plenum, New York, NY.

Hansel, W. and B.J. Weir, eds. 1990. Genetic Engineering of Animals. 240 pp. J. Reprod. Fertil. Suppl. No. 41.

Johnson, L.A., Flook, J.P. and H.W. Hawk. 1989. Sex preselection in rabbits: live births from $\mathrm{X}$ and $\mathrm{Y}$ sperm separated by DNA and cell sorting. Biol. Reprod. 41:199-203.

Juskevich, J.C. and C.G. Guyer. 1990. Bovine growth hormone: human food safety evaluation. Science 249:875-884.

Seidel, G.E. Jr., ed. 1985. Technology in Animal Agriculture: A Seminar for Investment Strategists. 337 pp. Colorado State University, Fort Collins, $\mathrm{CO}$.

Seidel, G.E., Jr. and R.P. Elsden. 1989. Embryo Technology. 101 pp. W.D. Hoard \& Sons, Fort Atkinson, WI. 